this molecule will have important implications for understanding diseases characterized by inappropriate $\mathrm{T}$ cell activation, including autoimmune diseases. To this end, however, we have a long way to go.

Address correspondence to: James P. Allison, Howard Hughes Medical Institute, Memorial Sloan-Kettering Cancer Center, 415 E. 68th Street, Box 470, New York, New York 10021, USA. Phone: (646) 888-2332; Fax: (646) 422-0470; E-mail: allisonj@mskcc.org.

1. Korman, A.J., Peggs, K.S., and Allison, J.P. 2006 Checkpoint blockade in cancer immunotherapy. Adv. Immunol. 90:297-339.

2. Peggs, K.S., Quezada, S.A., Korman, A.J., and Allison, J.P. 2006. Principles and use of anti-CTLA4 antibody in human cancer immunotherapy. Curr. Opin. Immunol. 18:206-213.

3. Okazaki, T., and Honjo, T. 2006. The PD-1-PD-L pathway in immunological tolerance. Trends Immunol.
27:195-201.

4. Barber, D.L., et al. 2006. Restoring function in exhausted CD8 T cells during chronic viral infection. Nature. 439:682-687.

5. Vogt, L., et al. 2006. VSIG4, a B7 family-related protein, is a negative regulator of $\mathrm{T}$ cell activation. J. Clin. Invest. 116:2817-2826. doi:10.1172/ JCI25673.

6. Langnaese, K., Colleaux, L., Kloos, D.U., Fontes, M., and Wieacker, P. 2000. Cloning of Z39Ig, a novel gene with immunoglobulin-like domains located on human chromosome X. Biochim. Biophys. Acta. 1492:522-525.

7. Helmy, K.Y., et al. 2006. CRIg: a macrophage complement receptor required for phagocytosis of circulating pathogens. Cell. 124:915-927.

8. Kim, J.K., et al. 2005. Characterization of monoclonal antibody specific to the Z39Ig protein, a member of immunoglobulin superfamily. Immunol. Lett. 99:153-161.

9. Holers, V.M., Kinoshita, T., and Molina, H. 1992. The evolution of mouse and human complement C3-binding proteins: divergence of form but conservation of function. Immunol. Today. 13:231-236.

10. Roozendaal, R., and Carroll, M.C. 2006. Emerging patterns in complement-mediated pathogen recognition. Cell. 125:29-32.

11. Gray, J.D., and Horwitz, D.A. 1988. Lymphocytes expressing type 3 complement receptors proliferate in response to interleukin 2 and are the precursors of lymphokine-activated killer cells. J. Clin. Invest. 81:1247-1254.

12. Claesson, M.H., and Miller, R.G. 1985. Functional heterogeneity in allospecific cytotoxic $\mathrm{T}$ lymphocyte clones. II. Development of syngeneic cytotoxicity in the absence of specific antigenic stimulation. J. Immunol. 134:684-690.

13. Yaskanin, D.D., Thompson, L.F., and Waxman, F.J. 1992. Distribution and quantitative expression of the complement receptor type 1 (CR1) on human peripheral blood T lymphocytes. Cell. Immunol. 142:159-176.

14. Wagner, C., et al. 2006. The complement receptor 1 , CR1 (CD35), mediates inhibitory signals in human T-lymphocytes. Mol. Immunol. 43:643-651.

15. Hong, K., et al. 1991. Reconstitution of C5 convertase of the alternative complement pathway with isolated C3b dimer and factors B and D. J. Immunol. 146:1868-1873

16. Brown, E.J. 1991. Complement receptors and phagocytosis. Curr. Opin. Immunol. 3:76-82.

\title{
Children are not little adults: just ask their hematopoietic stem cells
}

\author{
David A. Williams, ${ }^{1}$ Haiming $X u,{ }^{1}$ and Jose A. Cancelas ${ }^{1,2}$
}

1Division of Experimental Hematology, Cincinnati Children's Research Foundation and Cincinnati Children's Hospital Medical Center, Cincinnati, Ohio, USA. ${ }^{2}$ Hoxworth Blood Center, University of Cincinnati Academic Health Center, Cincinnati, Ohio, USA.

HSCs differ during ontogeny in some important parameters, including anatomic site of residence and cell cycling characteristics. In this issue of the JCI, Bowie et al. show that postnatal HSCs as well as fetal liver HSCs in mice are active in the cell cycle at much higher rates than that of adult HSCs; however, this increased frequency of cycling abruptly ceases 4 weeks after birth (see the related article beginning on page 2808). The cycling postnatal HSCs expressed high levels of CXC chemokine ligand 12 (CXCL12, also known as stromal cell-derived factor 1 [SDF-1]), a chemokine previously implicated in stem cell trafficking to the marrow cavity and shown to be expressed by cells within the hematopoietic microenvironment. These cells also possessed an engraftment defect impeding reconstitution in irradiated recipient mice, which was reversible by pretransplant administration of antagonists of the CXCL12 receptor, CXCR4. Such agents are currently clinically available, suggesting that this approach could be used to improve stem cell transplantation and engraftment.

HSCs, similar to all stem cells, have 2 defining properties: the capacity for multilineage differentiation and the capacity to generate daughter stem cells with identical proliferative/differentiation capacity through a

Nonstandard abbreviations used: CXCL12, CXC chemokine ligand 12; CXCR4, CXC chemokine receptor 4.

Conflict of interest: The authors have declared that no conflict of interest exists.

Citation for this article: J. Clin. Invest. 116:2593-2596 (2006). doi:10.1172/JCI30083. self-renewal process. Thus, HSCs comprise a compartment of undifferentiated stem cells while continuously providing more differentiated progenitor cells that are the more proximate source of all circulating blood cells. Adult HSCs are largely quiescent. It is estimated that approximately $95 \%$ of HSCs responsible for engraftment in stem cell transplantation assays are in the $\mathrm{G}_{0}$ phase of the cell cycle $(1,2)$. These stem cells show a slow rate of turnover, while their progenitor progeny, the majority of which are actively cycling, are responsible for the vast majority of the cell amplification that is necessary for the production of billions of blood cells each day.

\section{Hematopoiesis changes during ontogeny}

The stem cell compartment expands enormously during fetal development and after HSC transplantation as well as in times of hematopoietic stress. This expansion is associated with a much higher frequency of cycling HSCs, especially during embryonic and fetal stages of development. The anatomic site of hematopoiesis is also distinct in the fetus versus the adult, with the major site of blood formation occurring successively in the yolk sac, the aorta-gonadmesonephros, and the fetal liver during embryonic development (1-3), while it is restricted to the bone marrow cavity in adults (and the spleen in mice) (4).

Along with these changes in anatomic location of blood formation, there are important differences in other aspects of hematopoiesis during development. For instance, embryonic (yolk sac-derived) red blood cells are nucleated, while fetal 
A Fetal liver HSCs

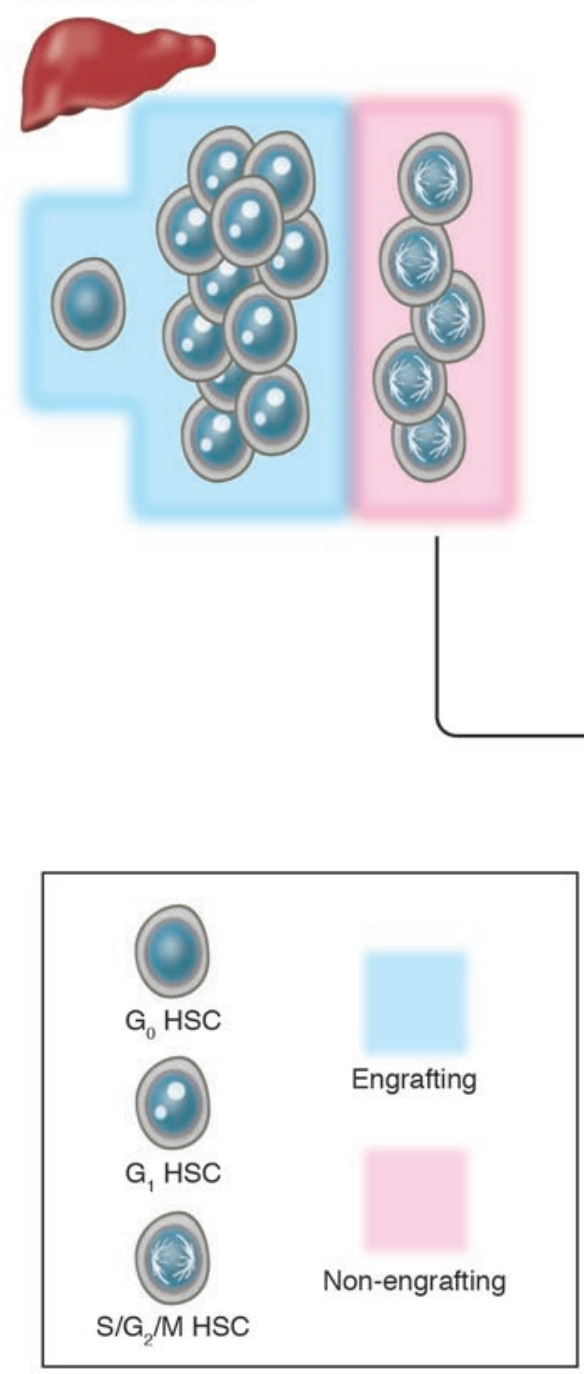

B Postnatal bone marrow-derived HSCs
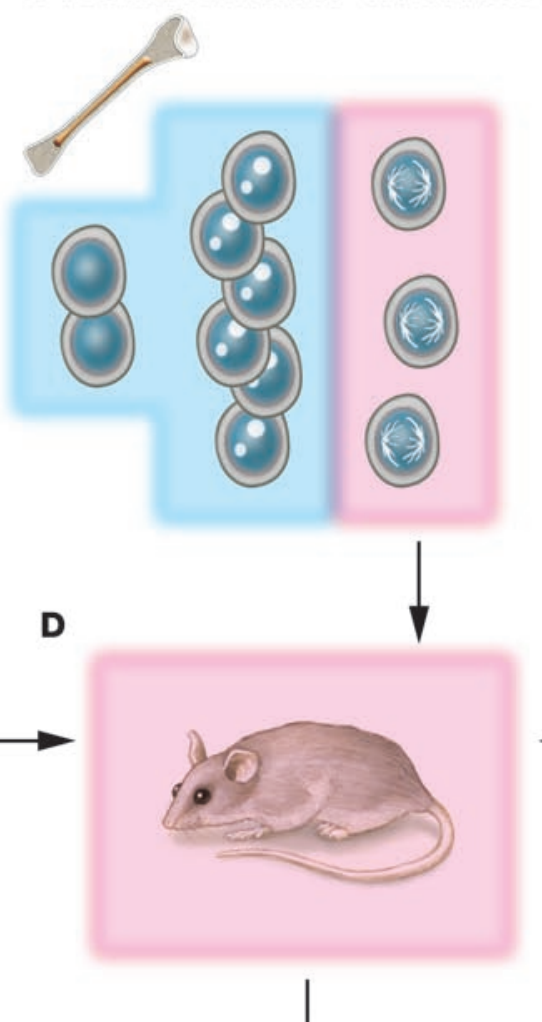

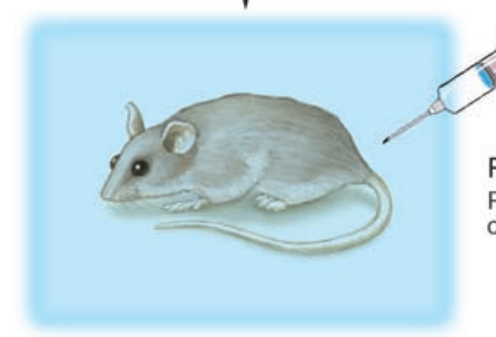

\section{Adult bone marrow-derived HSCs}

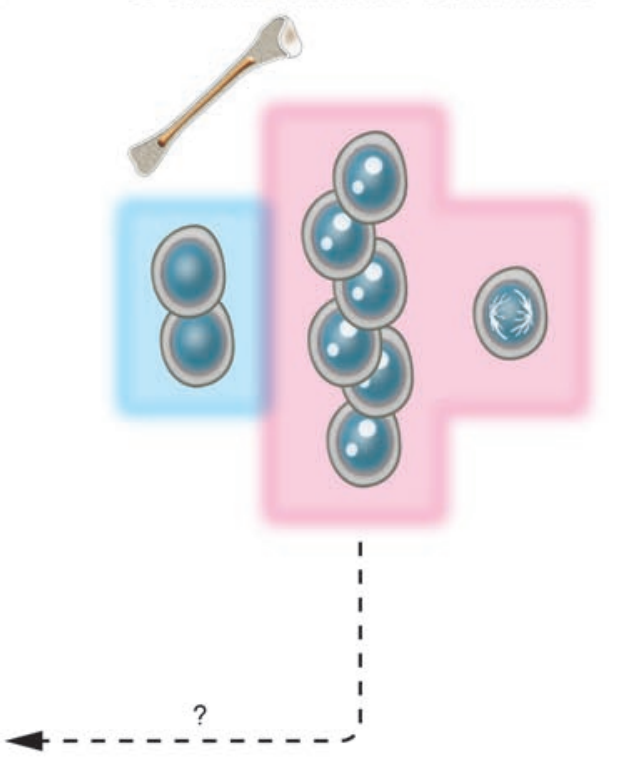

No engraftment

\section{Figure 1}

Content and engraftment ability of cycling HSCs derived from fetal liver and postnatal or adult bone marrow. (A) Fetal liver is enriched in $\mathrm{G}_{1} / \mathrm{S} /$ $\mathrm{G}_{2} / \mathrm{M}$ (cycling) HSCs. Fetal liver HSCs in the $\mathrm{G}_{1}$ phase are able to repopulate (blue background) syngeneic mice in a competitive repopulation assay. However, $\mathrm{S} / \mathrm{G}_{2} / \mathrm{M}$ HSCs have lost their repopulation potential (pink background). (B) HSC content of postnatal bone marrow is reduced compared with that of fetal liver, mostly due to a reduced $\mathrm{G}_{1}$ fraction, although most HSCs are in $\mathrm{G}_{1}$ phase. At the same time, postnatal bone marrow contains a higher frequency of $\mathrm{G}_{0}$ repopulating $\mathrm{HSC}$ s compared with fetal liver. (C) Most bone marrow-derived HSCs are in either $\mathrm{G}_{0}$ or $\mathrm{G}_{1}$ phase. The ability of HSCs in $\mathrm{G}_{1}$ phase to engraft to irradiated recipients is lost in adult bone marrow. (D) Administration of the CXCL12/ CXCR4 antagonist SDF-1G2 into recipient mice prior to transplantation enables $\mathrm{S} / \mathrm{G}_{2} / \mathrm{M}$ HSCs from fetal liver, postnatal bone marrow, and possibly (question mark) adult bone marrow to engraft in recipient mice.

liver-derived red blood cells are larger and express distinct antigens and different hemoglobin molecules compared with adult red blood cells. In addition, after birth, the production of new red blood cells is abruptly diminished, leading to a rapid reduction in bone marrow cellularity associated with a physiologic nadir in red blood cell count characteristically seen in the first postnatal month in humans. This physiological shift in erythropoiesis has been well studied (5). In this issue of the JCI, Bowie,
Eaves, and colleagues (6) report an abrupt change in the cycling of postnatal HSCs in mice reminiscent of this transition. The physiological relevance of this change is shown to have potential therapeutic implications for increasing HSC engraftment.

\section{Cycling status and repopulation ability of HSCs change during postnatal development}

What regulates the quiescence of HSCs is not completely known. There appears to be an exquisite regulation of the $G_{1}$ checkpoint in the cell cycle of these cells. Several exogenous as well as intrinsic, or cellautonomous, signals have been proposed to control cell proliferation of adult bone marrow-derived HSCs. Due to the changes in anatomic location of hematopoiesis and the presumed differences in the microenvironment within these hematopoietic locations, one might predict that the mechanisms controlling cell proliferation would vary depending on the developmental state. 
Indeed, unlike adult HSCs, fetal HSCs are continuously cycling, and this difference is likely to be controlled by either autonomous or microenvironment-dependent cues that differentially regulate the fate of HSCs in the embryo versus the adult. The molecular mechanisms involved, however, remain unclear.

Eaves's group and others (7-9) have previously reported that sorted, cycling (S/ $\mathrm{G}_{2} / \mathrm{M}$ phases) HSCs from adult mice do not home to the bone marrow and therefore cannot reconstitute hematopoiesis of lethally irradiated recipient mice. Bowie et al. (6) now provide additional insights into our understanding of cell-cycle activation of HSCs. By using in vivo thymidine-suicide methods in combination with careful measurements of bone marrow repopulation, they show in their current study that the vast majority of both fetal liver-derived and postnatal bone marrow-derived murine repopulating HSCs are in the $G_{1}$ cycling fraction of cells (Figure 1, A and B, and ref. 6). In contrast, and as shown by others (10), the vast majority of adult bone marrowderived repopulating HSCs are quiescent (in $\mathrm{G}_{0}$ phase; Figure $1 \mathrm{C}$ ) and therefore not susceptible to killing in thymidine-suicide assays. Bowie et al. also report a sudden and dramatic change in HSC proliferative activity that occurs in juvenile mice between 3 and 4 weeks of age (6). A surprising finding of the new study is that the cycling of HSCs changes abruptly well after the shift in HSC localization from the fetal liver to the bone marrow cavity. Thus, this change does not appear to be linked to the migration of HSCs during the last phases of embryogenesis, but rather is associated with the developmental status of the mouse, implicating the presence of stem cell-intrinsic regulation or age-dependent changes in the specific bone marrow microenvironment. In addition, this cycling activity of HSCs is associated with a reduced capacity of HSCs in $G_{1}$ to engraft into irradiated recipient mice (Figure 1C). While these results were expected based on previous observations of cycling adult bone marrow-derived HSCs, it has been previously shown that primitive buman fetal HSCs do repopulate immunodeficient (NOD/SCID) murine recipients, a widely accepted xenograft model of stem cell transplantation (11). Whether these disparate results represent species differences is not clear.

Bowie et al. (6) suggest a correlation of this abrupt change in cycling status and engraftment ability of postnatal hematopoiesis with that of specific telomere shortening in human children 5 years of age or older compared with infants or toddlers (12). This is of importance, since the maintenance of telomere length is crucial to cell self renewal, and HSCs express high levels of telomerase, a cellular reverse transcriptase that stabilizes telomere length (13). In addition, telomere length has been observed to decrease with repeated HSC transplantation (14), and HSCs from mice with targeted disruption of the telomere-maintenance gene telomerase reverse transcriptase undergo premature senescence (15).

\section{Blockade of the CXCL12/CXCR4 interaction reverses the lack of engrafting potential of $S / G_{2} / M$ HSCs}

Although several signaling pathways have been identified that affect the proliferation of HSCs, multiple attempts to expand the stem cell population in vitro have been largely unsuccessful. In part, this may reflect inadequate recapitulation of the microenvironment-associated cellcell interactions that were shown nearly 3 decades ago to be important for HSC fate (16). Thus, stem cell division in ex vivo systems that lack authentic hematopoietic microenvironmental cues is most often accompanied by proliferation that leads to stem cell differentiation. More recently, new strategies that lead to apparently improved HSC expansion ex vivo, albeit at generally modest levels, have been described (reviewed in ref. 17). These strategies include the use of more refined cytokine combinations using (that have) proteins favoring HSC proliferation with less differentiation; the blockading of signaling pathways such as TGF- $\beta$; the inhibition of cyclin-dependent kinase inhibitors p21Cip1/Waf1 and p27Kip1; the inhibition of dipeptidase CD26 or Rho GTPases; the enhanced expression or function of HoxB4, Notch, or $\beta$-catenin pathways; and the modification of the hematopoietic microenvironment by overexpression or infusion of osteoblast-acting parathormone.

In addition, Bowie et al. (6) show that expression of CXC chemokine ligand 12 (CXCL12, also referred to as stromal cellderived factor 1 [SDF-1]) is increased in HSCs during cell cycling. Lataillade et al. (18) previously suggested that HSC expression of CXCL12 suppressed apoptosis and promoted cell-cycle transition via an autocrine/paracine mechanism. CXCL12 is thought to act as a pivotal chemoattractant of HSCs through CXC chemokine recep- tor 4 (CXCR4) within the bone marrow microenvironment. Moreover, Bowie et al. show that pretransplant administration of SDF-1G2, an antagonist of CXCL12/ CXCR4 interaction, reversed the engraftment defect of HSCs in $S / G_{2} / M$ during transplantation (Figure 1 and ref. 6). These findings might suggest that the microlocalization of transplanted HSCs to specific bone marrow niches - which may be needed to activate a self-renewal program - depends on the strength of the CXCL12 gradient the cells encounter within the bone marrow space. Notably, this blockade of CXCL12/CXCR4 interaction by SDF$1 \mathrm{G} 2$ administered intravenously appears to act only in the peripheral blood, while sparing the bone marrow. The authors therefore speculate that the overexpression of CXCL12 by cycling HSCs may interfere in the response of HSCs to an intramedullary gradient of CXCL12 and thus prevent their self renewal, leading to differentiation, apoptosis, or sequestration of these cells in anatomic sites that do not support hematopoiesis. An intriguing possibility raised by the authors is that timed CXCR 4 signal blockade (for instance, by using the clinically available drug AMD3100) or an increase of CXCL12 levels in the medullary space may transiently restore an effective chemoattractant gradient for the HSCs in $\mathrm{S} / \mathrm{G}_{2} / \mathrm{M}$ and favor the lodging of HSCs otherwise insensitive to the CXCL12 gradient in the marrow microenvironment. If true, this approach would have important therapeutic applications to increase the efficiency of HSC engraftment in settings where the number and/or quality of these cells are limited. Examples of this include the use of umbilical cord blood for stem cell transplantation in adults and the engraftment of gene-corrected HSC populations following ex vivo manipulations required by current vector transduction protocols.

The findings described by Bowie et al. (6) that HSCs from adults differ from those of newborns open new areas of research aimed at defining the cell and molecular determinants of the change in cycling characteristics between postnatal and adult HSCs. One could speculate that the homing deficiency of bone marrow-derived $\mathrm{S} / \mathrm{G}_{2} / \mathrm{M}$ HSCs occurs at the time when weaning mice, equivalent to the childhood phases of the human lifespan, begin a rapid growth phase that ends in epiphysis closure and may be due to differences in the external cues associated with the osteogenesis developmental process. The hematopoietic 
changes reported by Bowie et al. may support a link between bone formation and hematopoiesis, which has been previously suggested by other authors $(19,20)$. Such a linkage could extend to the end of the lifespan, since progressive changes in both bone structure and marrow hematopoiesis activity continue from childhood to old age. The doors remain open for new, challenging research in this area.

Address correspondence to: David A. Williams, Division of Experimental Hematology, Cincinnati Children's Research Foundation and Cincinnati Children's Hospital Medical Center, 3333 Burnet Avenue, ML 7013, Cincinnati, Ohio 45229, USA. Phone: (513) 636-0364; Fax: (513) 636-3768; E-mail: david.williams@cchmc.org.

1. Fleming, W.H., et al. 1993. Functional heterogeneity is associated with the cell cycle status of murine hematopoietic stem cells. J. Cell Biol. 122:897-902.

2. Leemhuis, T., et al. 1996. Isolation of primitive human bone marrow hematopoietic progenitor cells using Hoechst 33342 and Rhodamine 123. Exp. Hematol. 24:1215-1224.

3. Palis, J., and Yoder, M.C. 2001. Yolk-sac hematopoiesis: the first blood cells of mouse and man. Exp.
Hematol. 29:927-936.

4. Wolber, F.M., et al. 2002. Roles of spleen and liver in development of the murine hematopoietic system. Exp. Hematol. 30:1010-1019.

5. Williams, D.A., Bunn, H.F., Sieff, C., and Zon, L. 2003. Hematopoiesis. In Blood:principles and practice of hematology. R.I. Handin, S.E. Lux, and T.P. Stossel, editors. Lippincott, Williams, \& Wilkins. 147-208.

6. Bowie, M.B., et al. 2006. Hematopoietic stem cells proliferate until after birth and show a reversible phase-specific engraftment defect. J. Clin. Invest. 116:2808-2816. doi:10.1172/JCI28310.

7. Glimm, H., Oh, I.H., and Eaves, C.J. 2000. Human hematopoietic stem cells stimulated to proliferate in vitro lose engraftment potential during their $\mathrm{S} / \mathrm{G}(2) / \mathrm{M}$ transit and do not reenter $\mathrm{G}(0)$. Blood. 96:4185-4193.

8. Orschell-Traycoff, C.M., et al. 2000. Homing and engraftment potential of Sca- $1(+) \operatorname{lin}(-)$ cells fractionated on the basis of adhesion molecule expression and position in cell cycle. Blood. 96:1380-1387.

9. Habibian, H.K., et al. 1998. The fluctuating phenotype of the lymphohematopoietic stem cell with cell cycle transit. J. Exp. Med. 188:393-398.

10. Passegue, E., Wagers, A.J., Giuriato, S., Anderson, W.C., and Weissman, I.L. 2005. Global analysis of proliferation and cell cycle gene expression in the regulation of hematopoietic stem and progenitor cell fates. J. Exp. Med. 202:1599-1611.

11. Wilpshaar, J., et al. 2002. Engraftment potential of human fetal hematopoietic cells in NOD/SCID mice is not restricted to mitotically quiescent cells. Blood. 100:120-127.

12. Rufer, N., et al. 1999. Telomere fluorescence mea- surements in granulocytes and T lymphocyte subsets point to a high turnover of hematopoietic stem cells and memory T cells in early childhood. J. Exp. Med. 190:157-167.

13. Morrison, S.J., Prowse, K.R., Ho, P., and Weissman, I.L. 1996. Telomerase activity in hematopoietic cells is associated with self-renewal potential. Immunity. 5:207-216.

14. Allsopp, R.C., Morin, G.B., DePinho, R., Harley, C.B., and Weissman, I.L. 2003. Telomerase is required to slow telomere shortening and extend replicative lifespan of HSCs during serial transplantation. Blood. 102:517-520.

15. Allsopp, R.C., et al. 2003. Effect of TERT overexpression on the long-term transplantation capacity of hematopoietic stem cells. Nat. Med. 9:369-371.

16. Dexter, T.M., Allen, T.D., Lajtha, L.G., Schoefield, R., and Lord, B.I. 1973. Stimulation of differentiation and proliferation of haemopoietic cells in vitro. J. Cell. Physiol. 83:461-474.

17. Attar, E.C., and Scadden, D.T. 2004. Regulation of hematopoietic stem cell growth. Lenkemia. 18:1760-1768.

18. Lataillade, J.J., et al. 2002. Stromal cell-derived factor 1 regulates primitive hematopoiesis by suppressing apoptosis and by promoting $\mathrm{G}(0) / \mathrm{G}(1)$ transition in CD34(+) cells: evidence for an autocrine/paracrine mechanism. Blood. 99:1117-1129.

19. Calvi, L.M., et al. 2003. Osteoblastic cells regulate the haematopoietic stem cell niche. Nature. 425:841-846.

20. Zhang, J., et al. 2003. Identification of the haematopoietic stem cell niche and control of the niche size. Nature. 425:836-841. 